\title{
ACTIVITY OF UPPER BODY MUSCLES IN DOUBLE POLING AND SKIERG WORKOUT
}

\author{
Roman Horyna ${ }^{1}$, Radka Bačáková ${ }^{1}$, Martina Chrástková ${ }^{1}$, Jan Sedlák ${ }^{2}$, \\ Roman Čmejla ${ }^{2}$, Bronislav Kračmar ${ }^{1}$ \\ Charles University in Praguel, Prague, Czech Republic \\ Czech Technical University in Prague ${ }^{2}$ Prague, Czech Republic
}

\begin{abstract}
Background. The aim of the study was to compare the involvement of upper body muscles during double poling and SkiErg Concept 2 workout and verify its specificity for cross-country skiing.

Methods. Ten elite Czech cross-country skiers performed double poling and SkiErg workout. Electromyography of selected upper body muscles and cycle characteristics were analysed. To monitor the electrical activity of muscles, we used the device ME6000. Data were analysed using Mega Win and MATLAB software version R2012b.

Results. Relative poling phase during double poling was $30.30 \pm 2.02 \%$ and during SkiErg workout $54 \pm$ $3.36 \%$. Pre-activation of trunk flexors was significantly higher during double poling due to high and forward body position before pole plant. Pre-activation of trunk flexors was not significantly different as pre-activation of shoulder and elbow extensors during SkiErg workout. Deactivation of these muscles came significantly later during SkiErg workout.

Conclusion. SkiErg cannot be considered a specific training method for cross-country skiing. It can be recommended to obtain specific power, but long-term application may cause disruption of double poling technique, especially timing of trunk flexors, shoulder and elbow extensors.
\end{abstract}

Keywords: cross country skiing, double poling, SkiErg, upper body, EMG.

\section{INTRODUCTION}

$r^{1}$ ross-country (XC) skiing is one of the most demanding endurance sports and displays a great variety and multiplicity of performance determinants. There are two basic skiing techniques, the classic style and skating style. Important technique of the classic style is double poling (DP). DP is mainly used on flat and slightly downhill and steep sections of the track, which is achieved at high speed locomotion (Fabre, Balestreri, Leonardi, \& Schena, 2010). The importance of DP has increased during the last two decades. It is not only used for mass starts and sprints, but also for long distance (Saltin, 1997; Stöggl, Lindinger, \& Müller, 2006). This technique put more emphasis on upper body muscles (Holmberg, Lindinger, Stöggl, Eitzlmair, \& Müller,
2005), which requires the necessary level of their specific strength. Cross-country skiers use a wide range of imitation drills due to the absence of snow in summer and sometimes also in winter. SkiErg Concept 2 (SkiErg) is often used for imitation drills in training (Figure 1). SkiErg workout is part of the test battery of the Czech national team in $\mathrm{XC}$ skiing. Imitation drills on SkiErg are ranked among semi-specific training methods, as well as roller skiing and other imitation drills (Hottenrot \& Urban, 2004).

Several studies have been performed to investigate physiological aspects (Hoffman et al., 1998; Saltin, 1997; Staib, Im, Caldwell, \& Rundell, 2000), fewer studies - kinematic and kinetic aspects (Hoffman et al., 1995; Holmberg 


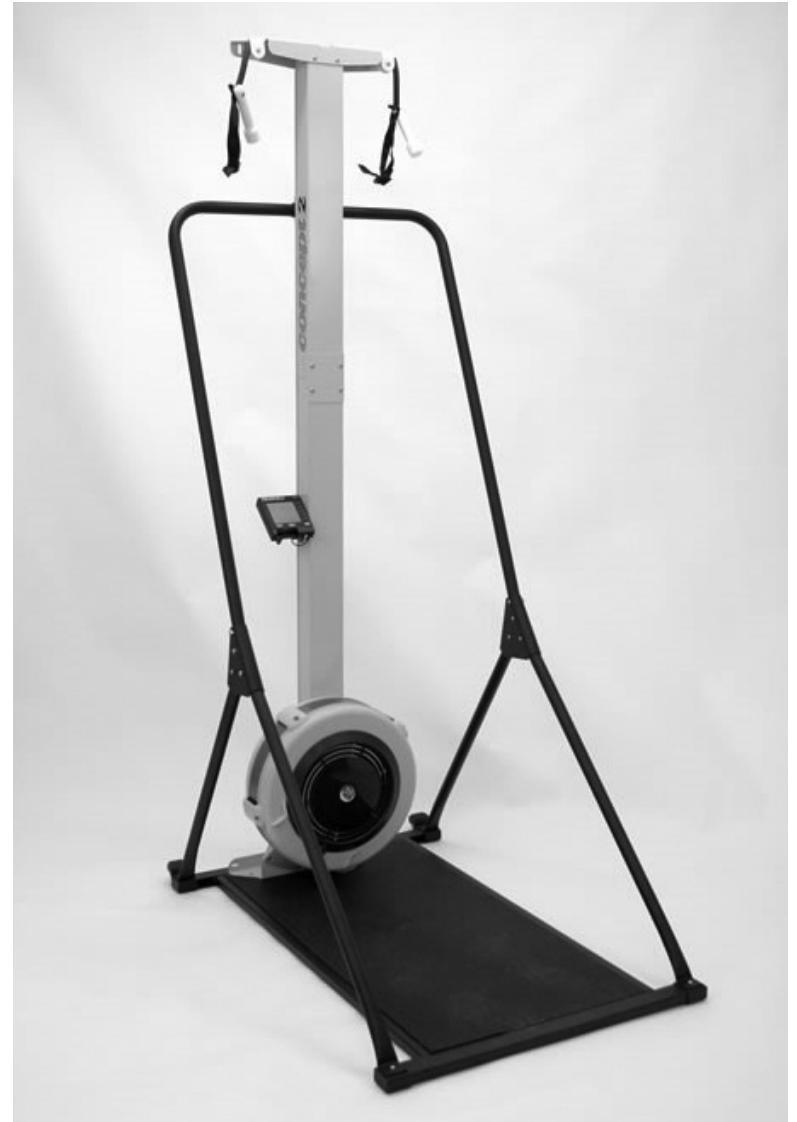

Figure 1. SkiErg "Concept 2"

et al., 2005; Horyna, Finková, \& Kračmar, 2012; Millet, Hoffman, Candau, \& Clifford, 1998; Smith, Fewster, \& Braudt, 1996) and very few studieskinesiological aspects (Chrástková, Bačáková, Špulák, Kračmar, \& Čmejla, 2012; Chrastkova, Bacakova, Spulak, Cmejla, \& Kracmar, 2013; Holmberg et al., 2005; Horyna, Bačáková, Špulák, Kračmar, \& Čmejla, 2014; Suchý \& Kračmar, 2008; Zoppirolli et al., 2013) of the DP technique. Holmberg et al. (2005) showed that muscles were engaged in sequential order starting with trunk and hip flexors, followed by shoulder extensors and the elbow extensor triceps brachii during DP. They found two DP strategies with different kinematic, kinetic and kinesiological characteristics. Nilsson, Tinmark, Halvorsen, and Arndt (2013) found a similar change in electromyography (EMG) activity while increasing the speed of locomotion or increasing the horizontal resistance during DP. Zoppirolli et al. (2013) suggest that the stretchshortening cycling effectiveness of the triceps brachii and latissimus dorsi muscles is a major determinant of DP performance.

So far, no study compared the kinesiology aspects and timing activation of relevant muscles during SkiErg workout with DP. Hottenrot and
Urban (2004) considered SkiErg as a semi-specific training method, but it was not supported by any biomechanical or kinesiological studies. The aim of the study was to compare the involvement of the upper body muscles during DP and SkiErg workout. Comparing both types of locomotion can clarify the intra-individual level of coordination similarity or difference and specificity of the SkiErg for XC skiing training.

\section{METHODS}

Subjects. Ten elite male Czech XC skiers, 18 \pm 1.1 years old, $179 \pm 6.1 \mathrm{~cm}$, and $68.5 \pm 5.8 \mathrm{~kg}$ volunteered to participate. All subjects were familiar with SkiErg workout, both as part of their training and in testing. They had a classical pole length $85 \pm 2 \%$ of body height. All the skiers were fully acquainted with the nature of the study before they gave their written informed consent to participate. The research techniques and protocol were approved by the Ethics Committee of Charles University, Prague, Czech Republic. Parental permission of young athletes under 18 years old is available at the authors upon authorization of the Ethics Committee.

Overall design. Intra-individual comparative analytical study investigated two types of locomotion: DP and SkiErg workout. DP was performed once on a $150 \mathrm{~m}$ section of the track with a slight incline of $1^{\circ}$. We analysed 20 cycles for each subject. Probands used his own XC skis adequately prepared for the snow conditions. DP and SkiErg workout were performed with the same intensity at the anaerobic threshold ( $80-85 \%$ of maximum heart rate). Probands have experience with this pace during training. SkiErg drag factor was chosen the same as in testing. For the evaluation of the measured data, the period was selected where the motion stereotype was stabilized. We observed two variables: cycle duration and muscle activity (timing).

EMG measurements. For kinesiological analysis we used EMG method which is noninvasive (De Luca, 1997). EMG measurement was completed with synchronized video recording using two cameras: SONY HDR-SR12 with a maximum video resolution of $1920 \times 1080$ pixels and frame rate 25 frames per second. For EMG recording we used a mobile device - ME 6000 Biomonitor (Mega Electronics, Kuopio, Finland) with sampling frequency $1000 \mathrm{~Hz}$. This device was carried on athlete's body. $\mathrm{Ag} / \mathrm{AgCl}$ electrodes 
(Medico Lead) were positioned on the belly of each muscle, longitudinally with respect to the underlying muscle fibres, in accordance with standard recommendations.

Electrodes were placed on the following muscles: $m$. obliquus externus abdominis $d x$ (OBLe); $m$. rectus abdominis $d x$, pars superior (RA); $m$. pectoralis major $d x$, pars sternocostalis (PMa); m. triceps brachii $d x$, caput longum (TRI); and $m$. latissimus dorsi $d x(L D)$. Laterality was not an observed variable.

The obtained data were analysed using software Mega Win and Matlab. In cooperation with the Faculty of Electrical Engineering of the Czech Technical University in Prague we created an original algorithm for the evaluation of the EMG data.

Each channel of raw EMG signal was individually high-pass filtered $(20 \mathrm{~Hz}$, Butterworth, 6 -th order) for the elimination of artefacts. Further the EMG signals were rectified and filtered by down-pass filter $(20 \mathrm{~Hz}$, Butterworth, 6-th order) for calculation of linear EMG envelope. The cutoff frequency $20 \mathrm{~Hz}$ of down-pass filter was used due to the dynamic of movement and accuracy of detection muscle activity in linear envelope. Lower value of cut-off frequency reduced time accuracy of muscle activity detection. The cut-off frequency increase does not improve the detection accuracy and reduces the robustness of detection algorithms.

The boundaries of movement cycles used for the calculation of average EMG envelopes are defined as time positions of consecutive local maxims in manually selected EMG channel. For this purpose, channels with specific EMG activity character are preferred. In each multi-channel EMG record, a channel with one significant peak in movement cycle was selected for the identification of movement cycle boundaries. The identified boundaries of individual movement cycles are used for linear envelope signal segmentation. Signal segments are linearly interpolated to uniform length 1000 points which correspond to 1 second with used sample frequency and are sufficient to the characterization of EMG average envelopes.

The EMG activity detection, respectively the timing of onsets and cessations of muscle contractions identification, is based on thresholding of EMG envelope signal, analogically applied in other research (De Luca, 1997; Hug \& Dorel, 2009; Konrad, 2005). The improved threshold algorithm is used for EMG activity detection and is described in detail in the research of Špulák, Čmejla, Bačáková, Kračmar, and Satrapová (2014). The EMG average envelope is utilized in order to improve detection results in combination with threshold detector used for each EMG channel separately. The threshold is set to $25 \%$ of difference between peak and minimum envelope value in relevant movement cycle.

The detected EMG activity with synchronously recorded video is obtained. The ground contact and highest position of the hand timing is identified in the video. One DP cycle was defined as the period from the start of the pole ground contact to the start of the subsequent pole ground contact, during SkiErg workout from the highest position of the hand to the subsequent highest position of the hand. This process was used for time normalization to $0-100 \%$ scale of DP cycle. The EMG activity is converted to relative scale and timing in several DP cycles is analysed.

The study is focused on the involvement of upper body muscles during two types of locomotion. We observed:

a) pre-activation as the average time between the moment of activation of selected muscles and the moment of pole ground contact (respectively the highest position of the hand during SkiErg workout).

b) post-activation as the average time between the moment of pole ground contact (respectively the highest position of the hand during SkiErg workout) and the moment of deactivation of selected muscles.

Cycle time, absolute and relative poling times were determined for each DP and SkiErg workout cycle.

Statistics. A Shapiro-Wilk's test $(p>.05)$ and visual inspection of their histograms showed that all of the biomechanical values were approximately normally distributed for all types of locomotion. The data are presented as means \pm standard deviations. A one-way analysis of variance ANOVA was conducted to evaluate the influence of locomotion type on the parameters of interest. The independent variable, type of locomotion, involved two groups: double poling and SkiErg workout. Post hoc comparisons to evaluate pairwise differences among locomotion means were conducted with the use of Tukey HSD test.

All statistical analyses were performed using the SPSS 11.0 Software for Windows (SPSS Inc., Chicago, IL, USA) and statistical significance defined as an $\alpha$ value of .05 or less. 


\section{RESULTS}

Kinematic variables are shown in Table 1 . The cycle time was $0.99 \pm 0.13 \mathrm{~s}$ during DP and $1.19 \pm$ $0.15 \mathrm{~s}$ during SkiErg workout. The poling time was $0.30 \pm 0.02 \mathrm{~s}$ during DP and $0.64 \pm 0.04 \mathrm{~s}$ during SkiErg workout, corresponding to $30.30 \pm 2.02 \%$ and $53.8 \pm 3.36 \%$ of the cycle time. All variables showed a significant difference.

Table 2 shows the values of muscles activation, which are further illustrated graphically (Figure 2). The timeline is transferred from the absolute (s) to relative time (\%) due to comparison.
Table 1. Cycle and poling time characteristics of DP and SkiErg workout

Note. DP - double poling.

\begin{tabular}{|l|c|c|c|}
\hline \multicolumn{1}{|c|}{ Parameter } & DP & SkiErg & $p$-value \\
\hline Cycle time (s) & $0.99 \pm 0.13$ & $1.19 \pm 0.15$ & .004 \\
\hline Poling time (s) & $0.30 \pm 0.02$ & $0.64 \pm 0.04$ & .000 \\
\hline Relative poling time (\%) & $30.30 \pm 2.02$ & $53.8 \pm 3.36$ & .000 \\
\hline
\end{tabular}

Table 2. Average duration of muscle activity during DP and SkiErg workout (\% of average cycle duration)

Note. OBLe - obliquus externus abdominis; RA - rectus abdominis; PMa - pectoralis major; TRI - triceps brachii; LD - latissimus dorsi; DP double poling.

\begin{tabular}{|l|c|c|c|}
\hline \multicolumn{1}{|c|}{ Parameter } & DP & SkiErg & $p$-value \\
\hline Pre-activation OBLe & $17.17 \pm 5.67$ & $7.27 \pm 4.90$ & .001 \\
\hline Post-activation OBLe & $18.75 \pm 5.68$ & $25.32 \pm 4.56$ & .032 \\
\hline Pre-activation RA & $17.3 \pm 5.09$ & $5.79 \pm 4.01$ & .000 \\
\hline Post-activation RA & $16.39 \pm 5.99$ & $25.21 \pm 4.22$ & .001 \\
\hline Pre-activation PMa & $5.15 \pm 4.51$ & $3.64 \pm 4.01$ & 1.000 \\
\hline Post-activation PMa & $20.61 \pm 7.64$ & $28.74 \pm 5.53$ & .028 \\
\hline Pre-activation TRI & $4.84 \pm 4.36$ & $2.01 \pm 2.36$ & .194 \\
\hline Post-activation TRI & $26.97 \pm 5.15$ & $33.08 \pm 4.53$ & .021 \\
\hline Pre-activation LD & $1.59 \pm 1.00$ & $1.77 \pm 1.22$ & 1.000 \\
\hline Post-activation LD & $26.53 \pm 5.85$ & $33.27 \pm 3.87$ & .009 \\
\hline
\end{tabular}

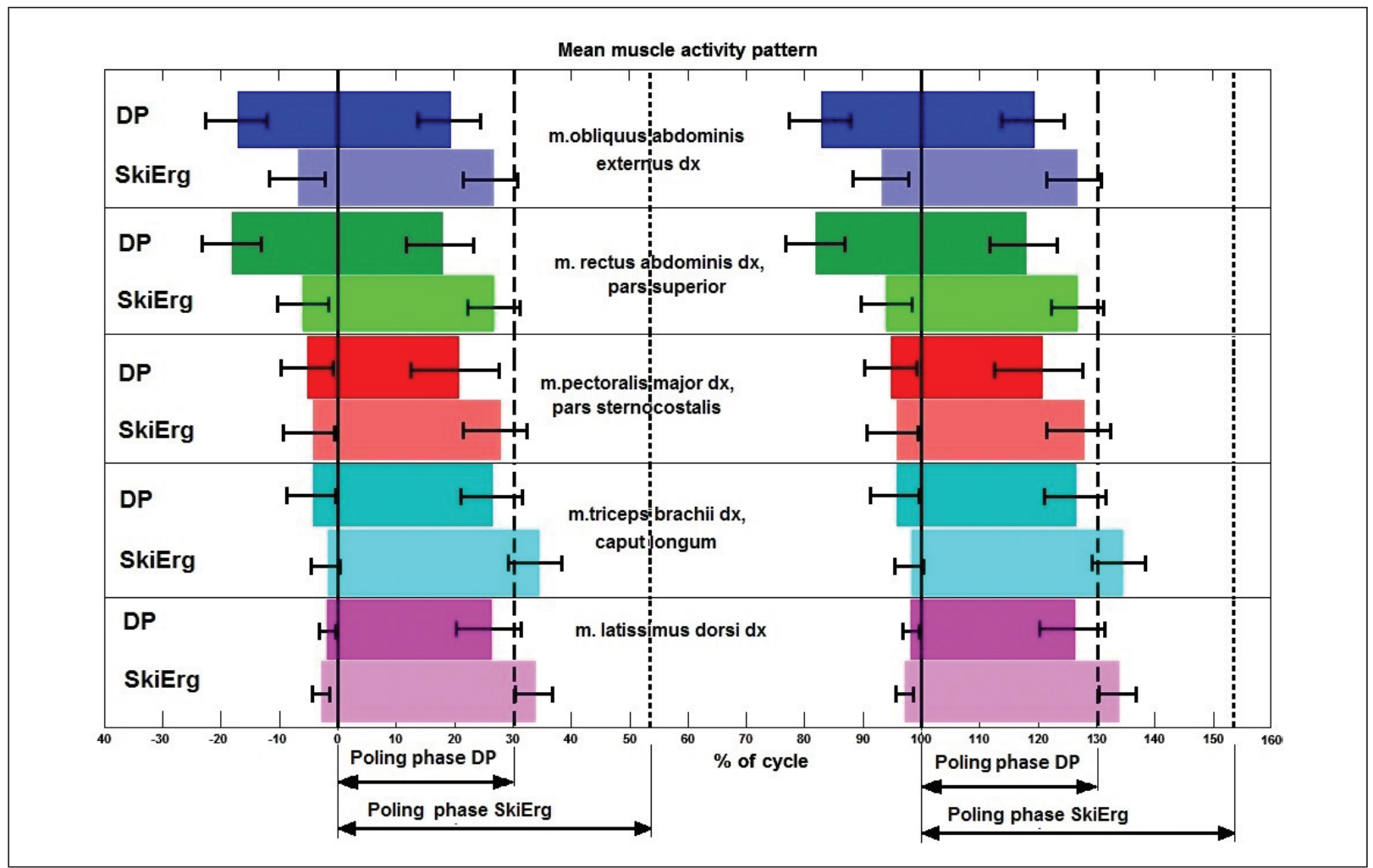

Figure 2. Relative activation time of selected muscles during DP and SkiErg workout in two average cycles

Note. The data are mean values. DP - double poling, m. - musculus, dx - dexter. 
The abdominal muscles reached considerable pre-activation during DP. OBLe (respectively RA) were activated $17.17 \pm 5.67 \%$ (respectively $17.3 \pm 5.09 \%$ ) before pole plant. Pre-activation of these muscles during SkiErg workout reached significantly lower values of $7.27 \pm 4.90 \%(5.79 \pm$ $4.01 \%$ ). They switched off according to a "first in, first out" pattern, $18.75 \pm 5.68 \%$ (respectively $16.39 \pm 5.99 \%)$ after pole plant during DP and $25.32 \pm 4.56 \%$ (respectively $25.21 \pm 4.22 \%$ ) during SkiErg workout.

Pre-activation of PMa as an antagonist with stabilizing function to $\mathrm{LD}$ was $5.15 \pm 4.51 \%$ during DP and $3.64 \pm 4.01 \%$ during SkiErg workout. The difference was not significant. PMa was switched off by $8.13 \%$ later during SkiErg workout than during DP.

Differences between activation of both propulsion muscles TRI and LD during both types of locomotion were not significant. Pre-activation reached values ranging from 1.59 to $4.84 \%$. TRI (respectively LD) was deactivated by $6.11 \%(6.74 \%)$ later during SkiErg workout in comparison to DP.

\section{DISCUSSION}

Average cycle time during DP was $0.99 \mathrm{~s}$, which corresponds to the study of Stöggl et al. (2006) with 1.01 s. Holmberg et al. (2005) found $1.13 \mathrm{~s}$, Nilsson et al. (2013) $1.075 \mathrm{~s}$ and Zoppirolli et al. (2013) $1.09 \mathrm{~s}$. The above mentioned authors conducted their measurements on the same level of intensity around the anaerobic threshold and the differences may be due to the nature of locomotion. They were measured under laboratory conditions on roller skis "in vitro". Average cycle time during SkiErg workout was 1.19 s, which is $20 \%$ longer compared with DP. It is due to longer poling time during SkiErg workout. Average poling time was $0.30 \mathrm{~s}$ during DP, which corresponds to the study Holmberg et al. (2005), which also indicate $0.30 \mathrm{~s}$. Nilsson et al. (2013) found $0.32 \mathrm{~s}$, Zoppirolli et al. (2013) found 0.31 s. Stöggl et al. (2006) found the average poling time of $0.51 \mathrm{~s}$ with inclination of $3^{\circ}$. We measured the poling time of $0.64 \mathrm{~s}$ during SkiErg workout, which is $113 \%$ longer compared with DP. There is a critical point during DP, where the hand with the pole reached the highest position with no angular velocity. The body is in a high starting position with distinctly extended hip, knee, and ankle joints and a clear forward shift of the body weight (forward lean). Then begins trunk flexion and angular velocity starts to increase followed by pole plant, in which the skier does not expend force from the zero level as it is during SkiErg workout, where forward lean position does not occur and propulsive phase begins at the moment of the highest position of the hand.

Coordination patterns (muscle sequencing) shown in Figure 2 point to the existence of muscle chains. The first one switched on trunk flexors (RA and OBLe). PMa starts to activate as the second and TRI with LD as the third. Pre-activation difference between the locomotion of these muscles is not significant and reaches a range from 0.18 to $2.83 \%$. They switched off according to a "first infirst out" pattern during DP, which corresponds to the measurements of Holmberg et al. (2005) and Horyna et al. (2014). The first was deactivated trunk flexors (RA and OBLe) followed by PMa, TRI and LD. Holmberg et al. (2005) found later deactivation of TRI compared to LD, which may be caused by longer cycle, when it is time to complete extension of the elbow joint. Zoppirolli et al. (2013) found the pre-activation of the TRI $4.3 \%$ during DP, which corresponds to our measurements (4.8\%). Preactivation of the LD was found $5.5 \%$, which does nott correspond to our data (1.59\%). Lower preactivation of the LD compared to TRI was found also by Nilsson et al. (2013). Holmberg et al. (2005) drew similar conclusions as Zoppirolli et al. (2013). They found longer pre-activation of LD compared to TRI. In the same order LD and TRI were deactivated. High activation of shoulder extensors PMa and LD was found by Holmberg et al. (2005). PMa has a double function with extension of the shoulder joint in the first part of poling phase, and a stabilizing function as antagonist to LD.

Significantly higher pre-activation of the trunk flexors RA and OBLe compared to other muscles was during DP. Earlier studies have focused on shoulder and elbow extensors without exploring possible important role of the abdominal muscles (Hoffman et al., 1995; Millet et al., 1998; Smith et al., 1996). Holmberg et al. (2005) and Nilsson et al. (2013) found a high level of EMG activity in RA and OBLe, indicating their important role in DP. They assume that this sequential pattern is involved in creating a low angle in the hip joint during poling, which leads to additional propulsive force.

Pre-activation of trunk flexors RA and OBLe is significantly lower during SkiErg workout due to the missing forward lean position. Trunk flexion occurs simultaneously at the moment of pulling handle of the SkiErg. Therefore the difference 
between pre-activation of trunk flexors and other muscles is not significant.

Deactivation of all selected muscles occurs 7-8\% later during SkiErg workout compared to DP. Prolonged post-activation of LD, TRI and PMa during SkiErg workout shows the consequences of an artificial instrument for simulating locomotion. The construction of the simulator does not allow creating the timing of poling as on the snow. Poling is longer, looser and its character is directed more towards the isokinetic contraction, while the propulsion effect during DP is based on the explosive muscular work. SkiErg workout has strength-endurance character, whereas DP has explosive-strength-endurance character.

Simultaneous activation timing of the abdominal muscles with other muscles shows again the artificial motion during SkiErg workout. The muscles in the muscle chains on the ventral side of the body do not create an optimal starting position (attitude) in which "punctum fixum" is formed for the work of the main propulsion muscle LD and the main antigravity muscle PMa (corresponding to the character of the movement during DP). Their coordination has the character of generalized movement without differentiation of muscle functions compared to DP with using trunk flexors for creating optimal situation for propulsion and antigravity muscles function of the shoulder girdle.

\section{CONCLUSION}

Muscle involvement during DP and SkiErg workout is different. Relative poling phase during DP is $30.3 \%$ with explosive-strength-endurance character of performance, while relative poling phase of SkiErg workout is $53.8 \%$ with isokinetic muscle contraction and strength-endurance character of performance.

Pre-activation of trunk flexors is significantly higher during DP due to forward lean position of the body, which is missing during SkiErg workout. The trunk flexors are involved in locomotion with minimal advance of the other propulsion muscles, which shows the artificial character of the movement with a lack of muscle function differentiation.

SkiErg cannot be considered as a specific training method for cross-country skiing. It can be recommended to obtain specific power, but longterm application may cause disruption of double poling technique, especially the timing of trunk flexors, shoulder and elbow extensors. Further investigation in this area should focus on kinetic and kinematic aspects when using DP and SkiErg workout.

Acknowledgement. The project was supported by SVV 2016-260346.

\section{REFERENCES}

Chrastkova, M., Bacakova, R., Spulak, D., Cmejla, R., \& Kracmar, B. (2013). The comparative analysis of free technique cross country and skating on roller skies. Studia Sportiva, 2, 95-101.

Chrástková, M., Bačáková, R., Špulák, D., Kračmar, B., \& Čmejla, R. (2012). Kineziologická analýza bruslení na kolečkových lyžích a na nordic blade. Studia Kinanthropologica, 13(3), 218-225.

Fabre, N., Balestreri, F., Leonardi, A., \& Schena, F. (2010). Racing performance and incremental double poling test on treadmill in elite female cross-country skiers. Journal of Strength and Conditioning Research, 24, 401-407. doi: 10.1519/JSC.0b013e3181c4d358

Hoffman, M. D., Clifford, P. S., \& Bender, F. (1995). Effect of velocity on cycle rate and length for three roller skiing techniques. Journal of Applied Biomechanics, 11, 257-266.

Hoffman, M. D., Clifford, P. S., Snyder, A., C., O’Hagan, K. P., Mittelstadt, S. W., Roberts, M., ... Gaskill, S. E. (1998). Physiological effects of technique and rolling resistance in uphill roller skiing. Medicine and Science in Sports and Exercise, 30, 311-317.
Holmberg, H. C., Lindinger, S. J., Stöggl, T. L., Eitzlmair, E., \& Müller, E. (2005). Biomechanical analysis of double poling in elite cross-country skiers. Medicine and Science in Sports and Exercise, 37, 807818. doi: 10.1249/01

Horyna, R., Bačáková, R., Špulák, D., Kračmar, B., \& Čmejla, R. (2014). Kineziologické aspekty soupažného běhu prostého a oboustranného bruslení jednodobého. Studia Kinanthropologica 15(3), 167-173.

Horyna, R., Finková, D., \& Kračmar, B. (2012). Současné pojetí soupažného běhu jednodobého. Studia Kinanthropologica, 13(3), 211-217.

Hottenrott, K., \& Urban, V. (2004). Das grosse Buch vom Skilanglauf. Aachen: Meyer \& Meyer.

Hug, F., \& Dorel, S. (2009). Electromyographicanalysis of pedaling: A review. Journal of electromyography and Kinesiology, 19, 182-198. doi: 10.1016/j. jelekin.2007.10.010

Konrad, P. (2005). The ABC of EMG - A Practical Introduction to Kinesiological electromyography. Retrieved from https://hermanwallace.com/download/ The_ABC_of_EMG_by_Peter_Konrad.pdf 
De Luca, C. J. (1997). The use of surface electromyography in biomechanics. Journal of Applied Biomechanics, 13(2), 135-163.

Millet, G. Y., Hoffman, M. D., Candau, R. B., \& Clifford, P. S. (1998). Poling forces during roller skiing: Effects of technique and speed. Medicine and Science in Sports and Exercise, 30, 1645-1653.

Nilsson, J., Tinmark, F., Halvorsen, K., \& Arndt, A. (2013). Kinematic, kinetic and electromyographic adaptation to speed and resistence in double poling cross-country skiing. European Journal of Applied Physiology, 113, 1385-1394.

Saltin, B. (1997). The physiology of competitive crosscountry skiing across a four decade perspective: with a note on training induced adaptations and role of training at medium altitude. In E. Müller, E. Kornexl, C. Raschner (Eds.), Science and Skiing (pp. 435-469). Cambridge: Chapman \& Hall.

Smith, G. A., Fewster, J. B., \& Braudt, S. M. (1996). Double poling kinematics and performance in crosscountry skiing. Journal of Applied Biomechanics, 12, 88-103.
Staib, J. L., Im, J., Caldwell, Z., \& Rundell, K. W. (2000). Cross-country ski racing performance predicted by aerobic and anaerobic double poling power. Journal of Strength and Conditioning Research, 14, 282-288.

Stöggl, T., Lindinger, S., \& Müller, E. (2006). Biomechanical validation of a specific upper body training and testing drill in cross-country skiing. Sports Biomechanics, 5, 23-46.

Suchý, J., \& Kračmar, B. (2008). Analysis of the kinesiology of skate skiing and roller skiing. Ugdymas. Kūno kultūra. Sportas, 3(70), 81-87.

Špulák, D., Čmejla, R., Bačáková, R., Kračmar, B., \& Satrapová, L. (2014). Muscle activity detection in electromyograms recorded during periodic movements. Computers in Biology and Medicine, 47(1), 93-103.

Zoppirolli, C., Holmberg, H. Ch., Pellegrini, B., Quaglia, D., Bortolan, L., \& Schena, F. (2013). The effectiveness of stretch-shortening cycling in upperlimb extensor muscles during elite cross-country skiing with the double poling technique. Journal of Electromyography and Kinesiology, 23, 1512-1519. 